\title{
SOME PROPERTIES OF ABSOLUTE RETURNS AS A PROXY FOR VOLATILITY
}

\author{
David E. Giles \\ Department of Economics, University of Victoria, \\ Victoria, B.C., Canada
}

August 2007

\begin{abstract}
We use the stochastic volatility model as a basis for investigating the statistical properties of absolute returns as a measure of latent volatility in financial markets. Our results are compared with existing results for squared returns.
\end{abstract}

Keywords: Volatility; stochastic volatility model; absolute returns; squared returns

JEL Classifications: C10, C46, G10

Proposed Running Head: Absolute returns as a proxy for volatility

\section{Author Contact:}

David Giles, Dept. of Economics, University of Victoria, P.O. Box 1700, STN CSC, Victoria, B.C., Canada V8W 2Y2; e-mail: dgiles@uvic.ca; FAX: (250) 721-6214 


\section{Introduction}

Various measures have been suggested as proxy variables for volatility in financial markets. The most popular are the squared returns and the absolute returns. For example, see Pagan and Schwert (1990), Rogers et al. (1994) and Ghysels et al. (2006) among others. ${ }^{1}$ Recently, Triacca (2007) has examined some of the properties of squared returns as an implicit estimator of the true unobserved volatility in a market. He uses the basic stochastic volatility (SV) model (e.g., Taylor, 1986), and a simple extension of this model to allow for a fat-tailed returns distribution, as the vehicle for his analysis. ${ }^{2}$ In this paper we undertake a similar analysis using absolute returns, rather than squared returns, as the volatility proxy. We also correct some errors in Triacca's results, and draw comparisons between the properties of these two estimators of volatility. Among our principal findings are that absolute returns are biased estimators of volatility, and where comparisons are possible, they result in higher mean squared error than when squared returns are used.

\section{Standard Stochastic Volatility Model}

The one-period return on an asset with price $p_{t}$ at time $t$ is defined as $r_{t}=\log _{e}\left(p_{t}\right)-\log _{e}\left(p_{t-1}\right)$, and the (unobserved) volatility in the returns is the latent variable $\sigma_{t}^{2} \equiv \operatorname{var}\left(r_{t} \mid I_{t-1}\right)$, where $I_{t-1}$ denotes the information set at time $t-1$. To make the volatility process specific, and using Triacca's (2007) notation for comparability, the basic stochastic volatility model (Taylor, 2005, pp. 278-83) can be used:

$$
\begin{aligned}
& r_{t}=\mu+\sigma_{t} z_{t} \quad ; \quad z_{t} \stackrel{i i d}{\sim} N(0,1) \\
& \log _{e}\left(\sigma_{t}^{2}\right)=\gamma_{0}+\gamma_{1} \log _{e}\left(\sigma_{t-1}^{2}\right)+u_{t} \quad ; \quad u_{t} \sim \stackrel{\text { iid }}{\sim} N\left(0, \sigma_{u}^{2}\right) \quad ; \quad\left|\gamma_{1}\right|<1
\end{aligned}
$$

where $u_{t}$ and $z_{t}$ are independently distributed. ${ }^{3}$ So, $\log _{e}\left(\sigma_{t}^{2}\right) \sim N\left[\gamma_{0} /\left(1-\gamma_{1}\right), \sigma_{u}^{2} /\left(1-\gamma_{1}^{2}\right)\right]$, given the stationarity condition in (2). The integer and fractional moments of $\sigma_{t}^{2}$ can be obtained using the result that if the random variable $Y \sim N(m, v)$, then $X=\exp (Y)$ is log-normally distributed with central moments given by

$$
E\left(X^{k}\right)=\exp \left(k m+k^{2} v / 2\right) .
$$

Following Taylor (2005, pp. 291-3) we can generalize the SV model so that (1) and (2) are augmented by the specification

$$
z_{t}=\zeta_{t} \sqrt{w_{t}} \quad ; \quad \zeta_{t}^{i i d} \sim N(0,1) \quad ; \quad(p-2) w_{t}^{-1} \sim \chi_{p}^{2} \quad ; \quad p>2
$$


and $\zeta_{t}$ is independent of $w_{t}$. Now $z_{t}$ is a Student-t variate, divided by its standard deviation, $\sqrt{p /(p-2)}$. For small values of $p$ this allows for a relatively fat-tailed unconditional distribution for the returns.

\section{Squared Returns as a Volatility Proxy}

Following Triacca (2007), we set $\mu=0$ in what follows. This is reasonable when prices are recorded daily, for example. If we use $r_{t}^{2}$ as an estimator of $\sigma_{t}^{2}$ it is readily shown that this estimator is unbiased (Triacca, 2007, p.256). Using (3) with $k=2$, its variance (and mean squared error), is

$$
\operatorname{MSE}\left(r_{t}^{2}\right)=E\left[\left(r_{t}^{2}-\sigma_{t}^{2}\right)^{2}\right]=2 \exp \left[\frac{2 \gamma_{0}}{1-\gamma_{1}}+\frac{2 \sigma_{u}^{2}}{1-\gamma_{1}^{2}}\right] .
$$

Under the Student-t specification, (4), $E\left(z_{t}^{2}\right)=1$, rather than $[p /(p-2)]$ as stated by Triacca (2007, p.256). So, $r_{t}^{2}$ is still an unbiased estimator of $\sigma_{t}^{2}$ in this more general Student-t case, contrary to Triacca's finding: $E\left(r_{t}^{2}-\sigma_{t}^{2}\right)=E\left(z_{t}^{2}-1\right) E\left(\sigma_{t}^{2}\right)=0$.

The even-order moments of a (non-standardized) Student-t variate, $T$, are:

$$
E\left(T^{k}\right)=p^{k / 2} \prod_{i=1}^{k / 2}\left[\frac{(2 i-1)}{(p-2 i)}\right] \quad ; \quad 0<k<p \quad(k \text { even }) \quad .
$$

Using (3) with $k=2$, and (6) with $k=4$, the correct expression for the variance (and MSE) of $r_{t}^{2}$ is also readily obtained:

$$
\operatorname{MSE}\left(r_{t}^{2}\right)=E\left[\left(r_{t}^{2}-\sigma_{t}^{2}\right)^{2}\right]=E\left(z_{t}^{4}-2 z_{t}^{2}+1\right) E\left(\sigma_{t}^{4}\right)=\frac{2(p-1)}{(p-4)} \exp \left[\frac{2 \gamma_{0}}{1-\gamma_{1}}+\frac{2 \sigma_{u}^{2}}{1-\gamma_{1}^{2}}\right] .
$$

From (5) and (7), for $p>4$ and any fixed values for the parameters, the MSE of $r_{t}^{2}$ as an estimator of $\sigma_{t}^{2}$ is greater under the Student-t specification than under the normality specification. As $p$ increases, the MSE decreases monotonically to that for the normal case.

\section{Absolute Returns as a Volatility Proxy}

We now extend the above discussion by using absolute returns rather than squared returns as the proxy for latent volatility. The latter is defined here in two ways - first as $\sigma_{t}$ (which is natural, given the implicit units of measurement); and second as $\sigma_{t}^{2}$, for reasons explained below. 


\section{Linear volatility}

Let us consider using $\left|r_{t}\right|$ as an estimator of $\sigma_{t}$. Using the properties of the integral representation of the gamma function, it is easily shown that the absolute moments of a standard normal variate, $Z$, are

$$
E\left(|Z|^{k}\right)=\frac{2^{k / 2}}{\sqrt{\pi}} \Gamma\left(\frac{k+1}{2}\right),
$$

and the corresponding expression in the (non-standardized) Student-t case is

$$
E\left(|T|^{k}\right)=\frac{p^{k / 2} \Gamma\left(\frac{k+1}{2}\right) \Gamma\left(\frac{p-k}{2}\right)}{\sqrt{\pi} \Gamma(p / 2)} ; p>k .
$$

Using these results we can obtain the bias and MSE of $\left|r_{t}\right|$, again with $\mu=0$. First, when $z_{t}$ is normally distributed, using (3) with $k=1 / 2$ and (8) with $k=1$,

$$
E\left(\left|r_{t}\right|-\sigma_{t}\right)=E\left(\left|z_{t}\right|-1\right) E\left(\sigma_{t}\right)=(\sqrt{2 / \pi}-1) \exp \left[\frac{\gamma_{0}}{2\left(1-\gamma_{1}\right)}+\frac{\sigma_{u}^{2}}{8\left(1-\gamma_{1}^{2}\right)}\right] \text {. }
$$

So, the estimator is downward-biased. Typical values for our parameters can be deduced from the evidence compiled by Taylor (2005, pp.287-8): $\gamma_{0} \in(0.95,0.99) ; \gamma_{1} \in(-0.54,-0.1)$ and $\sigma_{u}^{2} \in(0.0018,0.0478)$. Within these ranges the bias in (10) is negligible. Using (3) and (8) with $k$ $=1$,

$$
\operatorname{MSE}\left(\left|r_{t}\right|\right)=E\left[\left(\left|r_{t}\right|-\sigma_{t}\right)^{2}\right]=E\left(z_{t}^{2}-2\left|z_{t}\right|+1\right) E\left(\sigma_{t}^{2}\right)=2(1-\sqrt{2 / \pi}) \exp \left[\frac{\gamma_{0}}{1-\gamma_{1}}+\frac{\sigma_{u}^{2}}{2\left(1-\gamma_{1}^{2}\right)}\right] .
$$

When $z_{t}$ is a standardized Student-t variate, using (3) with $k=1 / 2$ and (9) with $k=1$,

$$
E\left(\left|r_{t}\right|-\sigma_{t}\right)=E\left(\left|z_{t}\right|-1\right) E\left(\sigma_{t}\right)=\left[\sqrt{\frac{p-2}{\pi}} \frac{\Gamma\left(\frac{p-1}{2}\right)}{\Gamma(p / 2)}-1\right] \exp \left[\frac{\gamma_{0}}{2\left(1-\gamma_{1}\right)}+\frac{\sigma_{u}^{2}}{8\left(1-\gamma_{1}^{2}\right)}\right] .
$$

So, for $p>1,\left|r_{t}\right|$ as an estimator of $\sigma_{t}$ is more negatively biased in the Student-t case than under normality. Similarly, from (3) and (9) with $k=1$,

$$
\operatorname{MSE}\left(\left|r_{t}\right|\right)=E\left[\left(\left|r_{t}\right|-\sigma_{t}\right)^{2}\right]=E\left(z_{t}^{2}-2\left|z_{t}\right|+1\right) E\left(\sigma_{t}^{2}\right)=2\left[1-\sqrt{\frac{p-2}{\pi}} \frac{\Gamma\left(\frac{p-1}{2}\right)}{\Gamma(p / 2)}\right] \exp \left[\frac{\gamma_{0}}{1-\gamma_{1}}+\frac{\sigma_{u}^{2}}{2\left(1-\gamma_{1}^{2}\right)}\right] \text {. }
$$

Comparing (11) and (13), for $p>2$, the MSE of $\left|r_{t}\right|$ as an estimator of $\sigma_{t}$ is greater when $z_{t}$ is Student-t distributed than when it is normally distributed. As $p$ increases, the MSE decreases 
monotonically to that for the normal case. This corresponds to the situation when $r_{t}^{2}$ is used as an estimator of $\sigma_{t}^{2}$.

\section{Quadratic volatility}

A less obvious possibility is to use $\left|r_{t}\right|$ as an estimator of $\sigma_{t}^{2}$, rather than $\sigma_{t}$. This is motivated by the empirical findings of Ghysels et al. (2006) and the theoretical results of Forsberg and Ghysels (2007), that absolute returns out-perform squared returns as a predictor of quadratic volatility. ${ }^{4}$ First, when $z_{t}$ is normally distributed. Using (3) with $k=1 / 2$ and 1 , and (8) with $k=1$,

$$
E\left(\left|r_{t}\right|-\sigma_{t}^{2}\right)=E\left(\left|z_{t}\right|\right) E\left(\sigma_{t}\right)-E\left(\sigma_{t}^{2}\right)=\sqrt{2 / \pi} \exp \left[\frac{\gamma_{0}}{2\left(1-\gamma_{1}\right)}+\frac{\sigma_{u}^{2}}{8\left(1-\gamma_{1}^{2}\right)}\right]-\exp \left[\frac{\gamma_{0}}{1-\gamma_{1}}+\frac{\sigma_{u}^{2}}{2\left(1-\gamma_{1}^{2}\right)}\right] \text {. }
$$

Similarly, using (3) with $k=1,3 / 2$ and 2 and (8) with $k=1$,

$$
\begin{aligned}
& M S E\left(\left|r_{t}\right|\right)=E\left[\left(\left|r_{t}\right|-\sigma_{t}^{2}\right)^{2}\right]=E\left(z_{t}^{2} \sigma_{t}^{2}-2 \sigma_{t}^{3}\left|z_{t}\right|+\sigma_{t}^{4}\right) \\
& =\exp \left[\frac{\gamma_{0}}{1-\gamma_{1}}+\frac{\sigma_{u}^{2}}{2\left(1-\gamma_{1}^{2}\right)}\right]-2 \sqrt{2 / \pi} \exp \left[\frac{3 \gamma_{0}}{2\left(1-\gamma_{1}\right)}+\frac{9 \sigma_{u}^{2}}{8\left(1-\gamma_{1}^{2}\right)}\right]+\exp \left[\frac{2 \gamma_{0}}{1-\gamma_{1}}+\frac{2 \sigma_{u}^{2}}{1-\gamma_{1}^{2}}\right]
\end{aligned}
$$

So, the sign and magnitude of this estimator's bias depend on the parameter values, as does the MSE for $\left|r_{t}\right|$ relative to that of $r_{t}^{2}$ as an estimator of $\sigma_{t}^{2}$. However, for the typical parameter value ranges noted above, (14) is always positive, and $\operatorname{MSE}\left(\left|r_{t}\right|\right)>\operatorname{MSE}\left(r_{t}^{2}\right)$.

When $z_{t}$ is a standardized Student-t variate:

$$
E\left(\left|r_{t}\right|-\sigma_{t}^{2}\right)=E\left(\left|z_{t}\right|\right) E\left(\sigma_{t}\right)-E\left(\sigma_{t}^{2}\right)=\left[\sqrt{\frac{p-2}{\pi}} \frac{\Gamma\left(\frac{p-1}{2}\right)}{\Gamma(p / 2)}\right] \exp \left[\frac{\gamma_{0}}{2\left(1-\gamma_{1}\right)}+\frac{\sigma_{u}^{2}}{8\left(1-\gamma_{1}^{2}\right)}\right]-\exp \left[\frac{\gamma_{0}}{1-\gamma_{1}}+\frac{\sigma_{u}^{2}}{2\left(1-\gamma_{1}^{2}\right)}\right]
$$

and

$$
\begin{aligned}
& \operatorname{MSE}\left(\left|r_{t}\right|\right)=E\left[\left(\left|r_{t}\right|-\sigma_{t}^{2}\right)^{2}\right]=E\left(z_{t}^{2} \sigma_{t}^{2}-2 \sigma_{t}^{3}\left|z_{t}\right|+\sigma_{t}^{4}\right) \\
& =\exp \left[\frac{\gamma_{0}}{1-\gamma_{1}}+\frac{\sigma_{u}^{2}}{2\left(1-\gamma_{1}^{2}\right)}\right]-2\left[\sqrt{\frac{p-2}{\pi}} \frac{\Gamma\left(\frac{p-1}{2}\right)}{\Gamma(p / 2)}\right] \exp \left[\frac{3 \gamma_{0}}{2\left(1-\gamma_{1}\right)}+\frac{9 \sigma_{u}^{2}}{8\left(1-\gamma_{1}^{2}\right)}\right]+\exp \left[\frac{2 \gamma_{0}}{1-\gamma_{1}}+\frac{2 \sigma_{u}^{2}}{1-\gamma_{1}^{2}}\right]
\end{aligned}
$$

Comparing (14) and (16), and (15) and (17), the effect of fatter tails in the distribution for $z_{t}$ is to reduce the bias but increase the MSE of the volatility proxy for any given set of parameter 
values. The second of these results accords with Triacca's (2007) (corrected) result when $r_{t}^{2}$ is used as a proxy for $\sigma_{t}^{2}$. Comparing (7) and (17) and simplifying, $\operatorname{MSE}\left(\left|r_{t}\right|\right)>\operatorname{MSE}\left(r_{t}^{2}\right)$ in the Student-t case iff

$$
\frac{(p+2)}{(p-4)} \exp \left[\frac{\gamma_{0}}{1-\gamma_{1}}+\frac{3 \sigma_{u}^{2}}{2\left(1-\gamma_{1}^{2}\right)}\right]+2\left[\sqrt{\frac{p-2}{\pi}} \frac{\Gamma\left(\frac{p-1}{2}\right)}{\Gamma(p / 2)}\right] \exp \left[\frac{2 \gamma_{0}}{1-\gamma_{1}}+\frac{5 \sigma_{u}^{2}}{8\left(1-\gamma_{1}^{2}\right)}\right]<1 .
$$

This condition is satisfied for the typical parameter ranges noted above and for all $p>4$.

\section{Conclusions}

Two popular proxies for the unobservable volatility of asset prices are the absolute and squared daily returns. Viewing these as alternative estimators of latent volatility, and using the stochastic volatility model as a vehicle, we have shown the following. For linear (quadratic) volatility, the absolute return is a downward (upward) biased estimator and the absolute value of this bias increases (decreases) as the tails of the returns distribution become thicker. In all of the situations

considered, allowing for fatter (than normal) tails in the returns distribution increases the MSE of both estimators. Finally, when estimating quadratic volatility, the absolute return has larger MSE than the squared return, at least for typical value of the parameters in the stochastic volatility model. This last result is somewhat surprising in light of the findings of Ghysels et al. (2006) and Forsberg and Ghysels (2007), and warrants further study. 


\section{References}

Forsberg, L. and Ghysels, E. (2007) Why do absolute returns predict volatility so well? Journal of Financial Econometrics, 5, 31-67.

Ghysels, E. Santa-Clara, P. and Valkanov, R. (2006) Predicting volatility: getting the most out of return data sampled at different frequencies, Journal of Econometrics, 131, 59-95.

Guégan, D. and Diebolt, J. (1994) Probabilistic properties of the $\beta$-arch model, Statistica Sinica, 4, 71-87.

Rogers, L. C. G., Satchell, S. E. and Yoon, Y. (1994) Estimating the volatility of stock prices: a comparison of methods that use high and low prices, Applied Financial Economics, 4, 241-47.

Taylor, ES. (1986) Modeling Financial Time Series, Wiley, New York, NY.

Taylor, S. J. (2005) Asset Price Dynamics, Volatility, and Prediction, Princeton University Press, Princeton, NJ.

Triacca, U. (2007) On the variance of the error associated to the squared return as proxy for volatility, Applied Financial Economics Letters, 3, 255-27. 


\section{Footnotes}

1. Other measures include the daily price range - e.g., Ghysels et al. (2006).

2. The appeal of the SV model over the GARCH model in this context is that it allows the time-dependence of volatility to be stochastic rather than deterministic.

3. The independence assumption can be relaxed without affecting the main results below if appropriate conditioning arguments are used.

4. In addition, Guégan and Diebolt (1994) consider the so-called $\beta$-ARCH models that include the special case of predicting future conditional variance through past absolute returns. 